\title{
Survie et performances de croissance des pintadeaux en milieu contrôlé au nord du Burkina Faso
}

\author{
R. SANFO ${ }^{1 *}$, S. OUOBA IMA $^{2}$, I. SALISSOU ${ }^{3}$ et H. H. TAMBOURA ${ }^{1}$ \\ ${ }^{1}$ Institut de l'Environnement et de Recherches Agricoles (INERA), Direction Régionale de l'Environnement \\ Agricole et de Formation de Kamboinsé, Ouagadougou, Burkina Faso. \\ ${ }^{2}$ Institut de l'Environnement et de Recherches Agricoles (INERA), Direction Régionale de l'Environnement \\ Agricole et de Formation, Station de Saria, Burkina Faso. \\ ${ }^{3}$ Institut National de la Recherche Agronomique du Niger (INRAN), Niger. \\ *Auteur correspondant ; E-mail : r_sanfo@yahoo.fr
}

\section{RESUME}

Au Burkina Faso, les pintadeaux connaissent une très forte mortalité liée à des causes multifactorielles, et leur vitesse de croissance reste encore méconnue par les techniciens, tant de la recherche que du développement. Cette étude vise à évaluer les performances de croissance et le taux de mortalité des pintadeaux dans les conditions d'élevage améliorées. Le maximum du poids des œufs incubés est compris dans la catégorie de 40-45 g. Les de mensurations des œufs ont révélé une longueur moyenne de 47,4 $\pm 1,428$ $\mathrm{mm}$ et 38,65 $\pm 1,08 \mathrm{~mm}$ en moyenne pour le grand diamètre. Le taux de mortalité a été de 9,10\%, 7,00\% et $9,80 \%$ respectivement chez le producteur I, II et III avec une moyenne de $8,63 \%$. Le poids vifs des pintadeaux à l'éclosion est de 26,59 $\pm 2,77$ g pour les deux sexes. Pour les femelles, il est de 27,69 $\pm 2,18 \mathrm{~g}$ et de 25,48 \pm 3,35 g pour les mâles. La courbe de croissance des femelles est largement au dessus de celle des mâles. Les pintadeaux femelles ont un GMQ de 2,84 $\mathrm{g}$ et les pintadeaux mâles de 2,27 $\mathrm{g}$ pendant les deux premières semaines. Le Gain Moyen Quotidien est de 3,78 g pendant les quatre mois. Le test révèle un effet positif du protocole sur la mortalité des pintadeaux.

(c) 2015 International Formulae Group. All rights reserved.

Mots-clés : Pintadeaux, mortalité, croissance, Burkina Faso.

\section{INTRODUCTION}

La pintade locale (Numida meleagris), suscite un intérêt de plus en plus grandissant dans la plupart des pays en développement. Ses produits sont à l'usage de la reproduction, de l'autoconsommation, des dons, des sacrifices et surtout la commercialisation (Sanfo et al., 2007). Au Burkina Faso, elle représente $19,2 \%$ de l'effectif des 30.940 .068 volailles, soit 6.117 .826 de sujets, et occupe ainsi le second rang après la poule $(76,3 \%)$ selon Kaboret et al. (2002).

Cette pintade est parfaitement adaptée aux conditions climatiques et aux contraintes alimentaires des zones à faible pluviométrie (MRA, 2003). Elle est créditée du statut d'espèce à doubles fins (viande, œufs) et jouit d'une rusticité remarquable. De part sa très grande prolificité et son cycle de reproduction court, elle favorise la reconstitution des effectifs après les épisodes mortels et les 
déficits pluviométriques très fréquents dans le pays. Les produits de la pintade locale sont par ailleurs particulièrement prisés par les populations rurales et urbaines et sont très abondant dans les différents marchés locaux.

Mais la méléagriculture (l'élevage de la pintade) connaît de très grandes difficultés, notamment celle de la mortalité des pintadeaux. Cette difficulté fait que, les efforts des producteurs restent sans résultats majeurs, car elle réduit la productivité numérique de cette pintade. Sanfo (2007) réussit à réduire cette mortalité à $6 \%$ en logeant les pintadeaux dans des éleveuses, en chauffant leur habitat et en apportant du saccharose dans leur eau de boisson en milieu contrôlé.

La présente étude a pour objectif d'évaluer le taux de mortalité et les performances de croissance des pintadeaux dans un système d'élevage purement intensif.

\section{MATERIEL ET METHODES}

\section{Présentation de la zone d'étude}

L'essai a été conduit dans la région du Sahel et dans province de Dori.

\section{Origine et calibrage des œufs}

Les œufs ont été approvisionnés par les producteurs de Dori, dans la région du Sahel du Burkina Faso, qui sont au nombre de trois. Chacun a apporté deux cent cinquante (250), soit un total de sept cent cinquante (750) œufs.

Ces œufs avaient une durée maximale de huit jours (8) par rapport au temps de leur ponte. Les mesures métriques ont été effectuées à l'aide d'un calibreur micrométrique électronique de marque $\mathrm{CE}$, d'une portée de $150 \mathrm{~mm}$ et d'une sensibilité de $0,01 \mathrm{~mm}$.

Les mesures pondérales ont été réalisées à l'aide d'une balance électronique, de marque AND, d'une portée de $180 \mathrm{~g}$ et d'une sensibilité de $0,1 \mathrm{mg}$. Elles ont été réalisées une fois toutes les deux semaines.

\section{Incubation des oufs}

Le calibrage a consisté à la pesée de même qu'à la détermination de la longueur et $\mathrm{du}$ grand diamètre selon la méthode de
(Gendron et Blentz, 1970). Cette opération a porté sur l'ensemble des œufs qui étaient de 845. Les œufs calibrés ont ensuite été répartis en catégories de poids d'un intervalle de $5 \mathrm{~g}$ suivant les normes de la CEE rapportées par (Gendron et Blentz, 1970). Une température moyenne de $37,8{ }^{\circ} \mathrm{C}$, une humidité relative moyenne de $42,4 \%$ et portée à $70 \%$ à l'éclosion. Un incubateur de marque Maino Enrico, de type 2-500 FH avec une capacité de 2.500 œufs a servi à l'incubation des œufs. L'éclosoir utilisé était de même marque, type et capacité que l'incubateur. Chacun de ces éléments est muni d'un système de ventilation forcée. Le retournement était manuel et réalisé trois fois par jour. Le taux d'éclosion a été de $80,00 \%$ pour le nombre total d'œufs mis à couver. Les pintadeaux ont été séparés en trois lots de deux cent vingt cinq (225) chacun, puis remis aux producteurs I, II, et III. Ils ont été soumis aux traitements suivants :

- Ils ont été logés dans des éleveuses qui sont des appareils de chauffage utilisé pour l'élevage des pintadeaux et destiné à maintenir une température conforme aux besoins des jeunes animaux ;

- Ils ont été nourris avec des aliments industriels de poulets à volonté (Tableau 1) ;

- Ils ont été réchauffés à l'aide de lampes tempêtes allumées pendant la durée de leur vie dans les éleveuses ;

- Du sucre, saccharose, a été dissout à 2,5\% dans l'eau de boisson apportée de façon ad libitum. Le sucre est un aliment énergétique facilement absorbable et aide ainsi les pintadeaux à lutter contre le froid ;

- Ils sont restés dans les éleveuses pendant quatre (4) mois.

\section{Analyse statistique}

Les analyses statistiques ont été réalisées à l'aide du logiciel Statistica version 6.0. La comparaison des moyennes a été faite au seuil de 5\% par le test t de Student.

\section{RESULTATS \\ Poids des oufs \\ Dans l'ensemble, le poids des œufs varie entre $25 \mathrm{~g}$ et $45 \mathrm{~g}$ avec $49 \%$ des œufs du}


sahel pesant entre 41 et 45 g et $49 \%$ des œufs originaires du Centre pesant entre 36 et $40 \mathrm{~g}$ (Figure 1). Les mensurations des œufs du Sahel et du Centre sont résumées dans le Tableau 2. La longueur moyenne des œufs est de $47,4 \pm 1,42 \mathrm{~mm}$ et le grand diamètre moyen est de $38,65 \pm 1,08 \mathrm{~mm}$.

\section{Taux de mortalité des pintadeaux}

Le taux de mortalité moyen a été de $8,63 \%$. Les taux de mortalités des pintadeaux ont été respectivement de $9,10 \%$ chez le producteur I, de $7,00 \%$ chez le producteur II et $9,80 \%$ chez le producteur III.

\section{Courbe de Gain Moyen Quotidien}

Les GMQ à deux semaines d'âge ont été de 2,84 g chez les femelles et de 2,27 g chez les mâles. Le GMQ moyen en quatre mois des pintadeaux a été de 3,78 g. Le GMQ moyen de 4,18 $\mathrm{g}$ des femelles est supérieur à
3,38 g enregistré chez des mâles. Ils sont confondus au démarrage, puis sont différenciés par la suite (Figure 2). De 2 à 4 mois, les GMQ des pintadeaux ont été de $5,00 \mathrm{~g}(4,50 \mathrm{~g}$ chez les mâles et $5,51 \mathrm{~g}$ chez les femelles). Dans l'ensemble, le dimorphisme est en faveur des femelles qui gagnent plus de poids que les mâles.

\section{Croissance pondérale des pintadeaux}

Le poids vif des pintadeaux est de $26,59 \pm 2,77 \mathrm{~g}$ pour les deux sexes. Pour les femelles, il est de $27,69 \pm 2,18 \mathrm{~g}$ et pour les mâles et de $25,48 \pm 3,35 \mathrm{~g}$. La courbe de croissance des pintadeaux montre que les femelles croissent plus rapidement que les mâles (Figure 3). Les poids des males et femelles sont confondus avant l'âge de quarante cinq (45) jours et se différencient audelà.

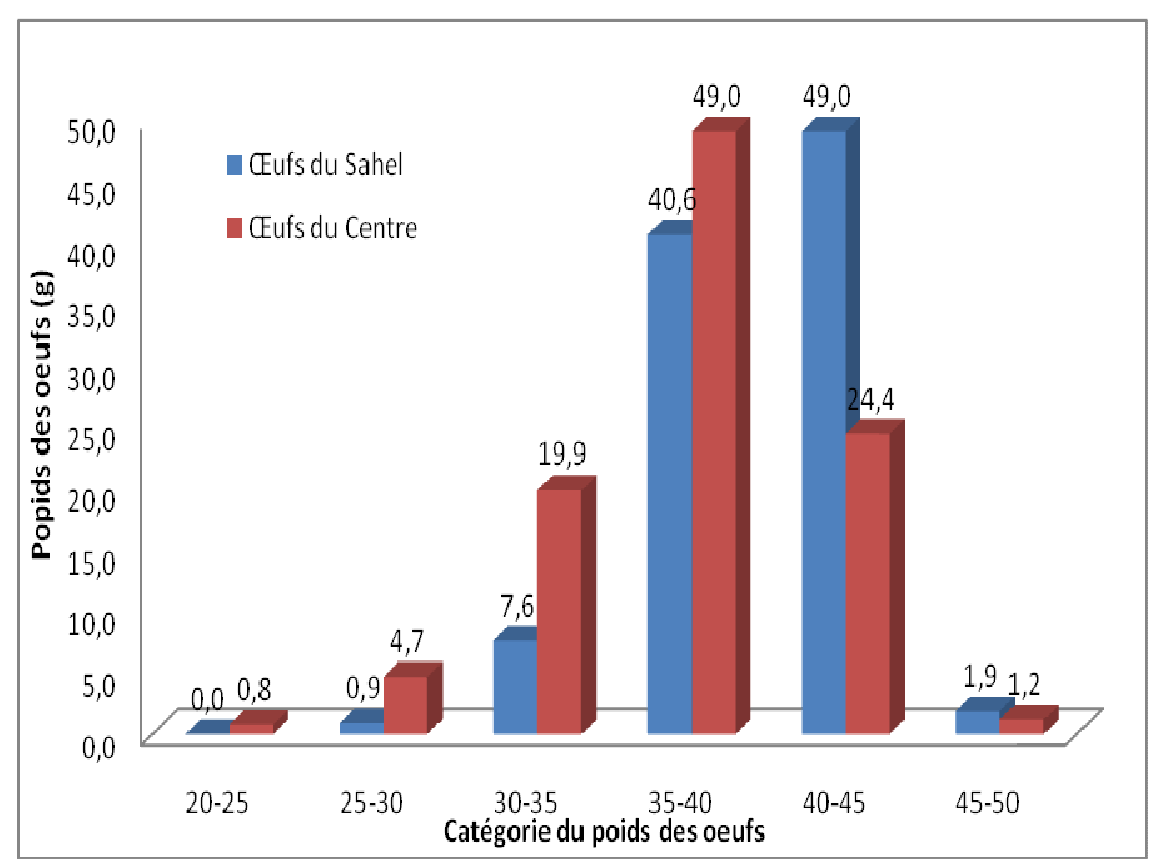

Figure 1: Comparaison des pourcentages du poids des œufs du Sahel et du Centre par catégorie 


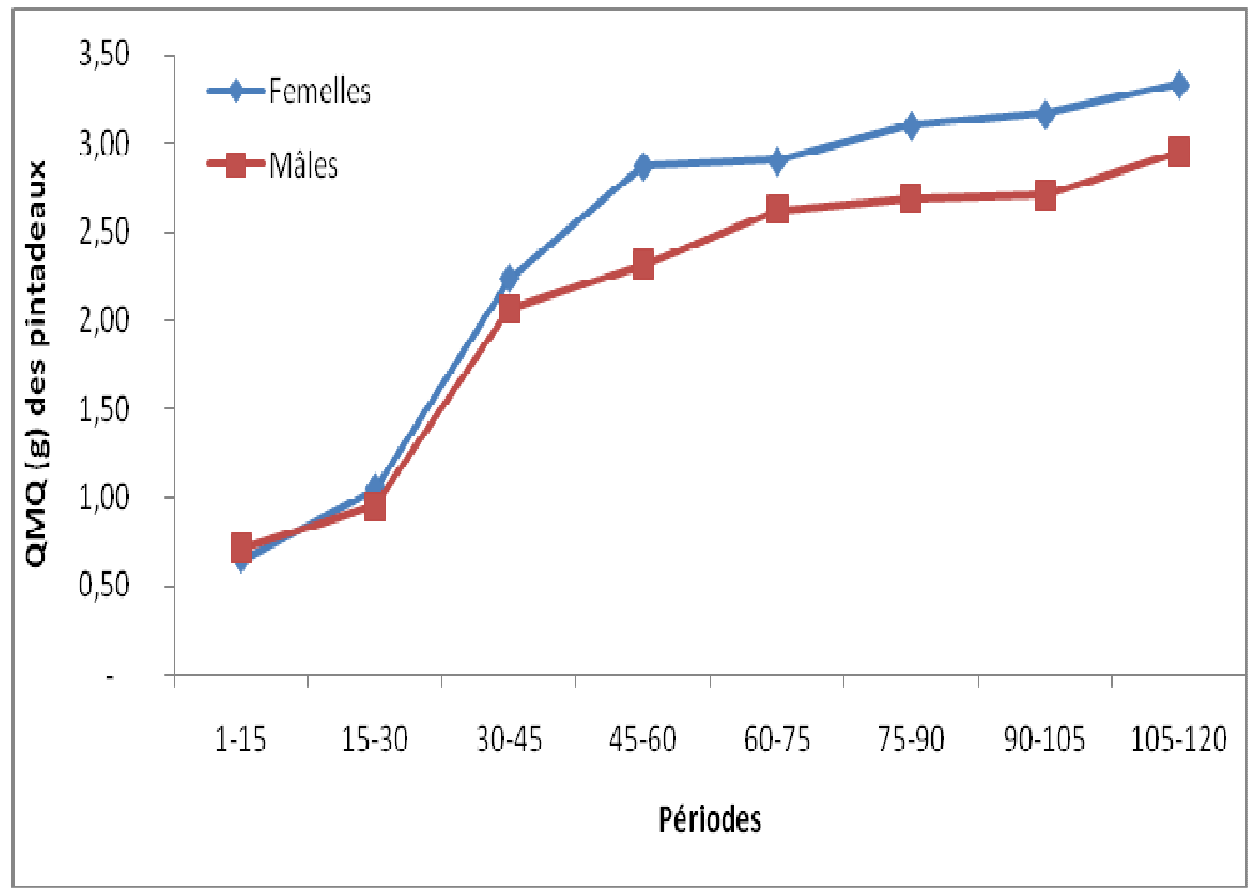

Figure 2 : Evolution des Gains Moyens Quotidiens (GMQ) des pintadeaux.

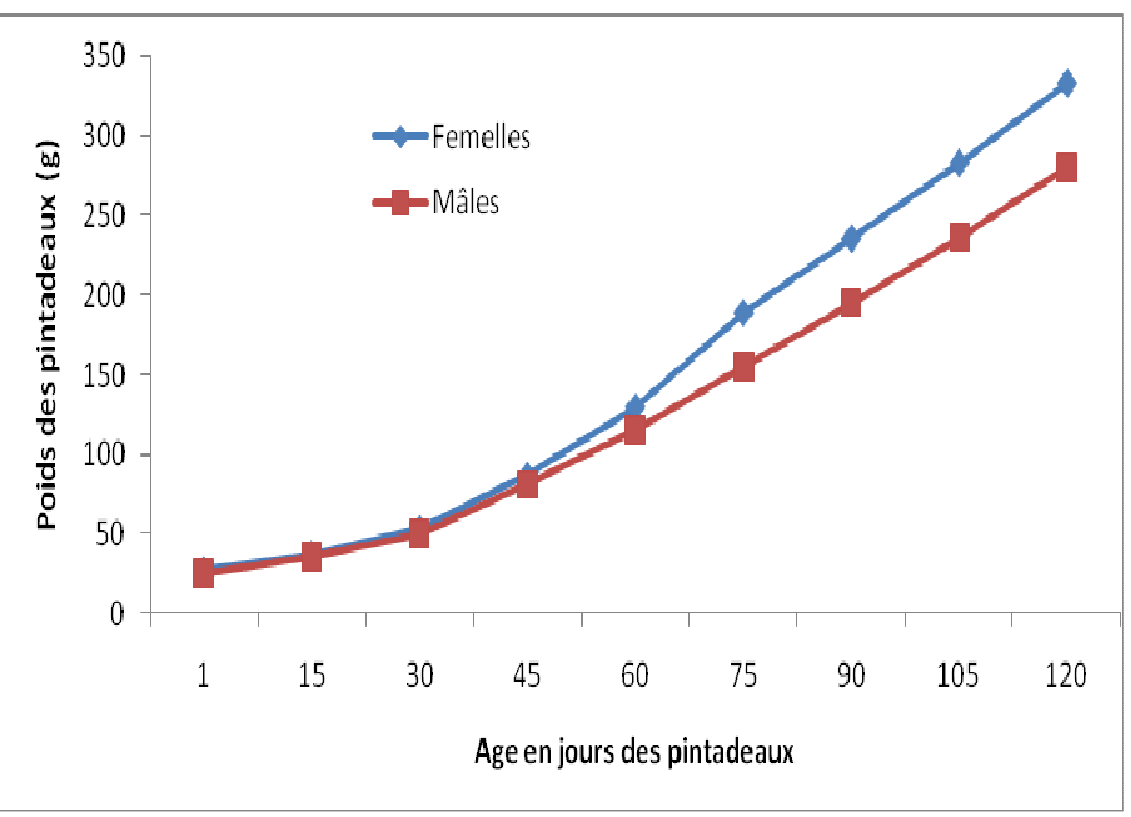

Figure 3 : Courbe de croissance pondérale des pintadeaux 
R. SANFO et al/Int. J. Biol. Chem. Sci. 9(2): 703-709, 2015

Tableau 1: Valeur bromatologique des aliments.

\begin{tabular}{|c|c|c|c|c|c|c|c|c|c|c|}
\hline Type d'aliment & $\mathbf{E}^{2}$ & MS & $\mathbf{P B}^{1}$ & MG $^{1}$ & $\mathbf{C B}^{1}$ & $\mathbf{C a}^{\mathbf{T}}$ & $\mathbf{P}^{1}$ & Lys $^{1}$ & Met $^{1}$ & Cys $^{1}$ \\
\hline Démarrage chair & 2970 & 89,5 & 19,8 & 4,4 & 4,0 & 1,1 & 0,7 & 1,1 & 0,5 & 0,3 \\
\hline Croissance & 2900 & 89,7 & 16,5 & 4,1 & 5,3 & 1,4 & 0,6 & 0,7 & 0,3 & 0,4 \\
\hline
\end{tabular}

${ }^{1}$ : En pourcent de la matière sèche, ${ }^{2}$ : en kcal

Tableau 2: Mesures métriques des œufs du Sahel et du Centre.

\begin{tabular}{lcccc}
\hline Classe de poids des œufs & \multicolumn{2}{c}{ Longueur $(\mathbf{m m})$} & \multicolumn{2}{c}{ Grand diamètre $(\mathbf{m m})$} \\
\hline & Sahel & Centre & Sahel & Centre \\
\hline $25-30$ & $44,53 \pm 2,04$ & $44,2^{\mathrm{a}} \pm 2,0$ & $37,27 \pm 2,21$ & $34,5^{\mathrm{a}} \pm 0,7$ \\
$30-35$ & $46,8 \pm 1,68$ & $45,8^{\mathrm{b}} \pm 1,8$ & $38,35 \pm 1,27$ & $36,2^{\mathrm{b}} \pm 1,2$ \\
$35-40$ & $48,33 \pm 1,13$ & $47,7^{\mathrm{c}} \pm 1,8$ & $38,58 \pm 0,62$ & $37,6^{\mathrm{c}} \pm 1,2$ \\
$40-45$ & $48,62 \pm 1,14$ & $49,5^{\mathrm{d}} \pm 1,9$ & $39,14 \pm 0,70$ & $39,0^{\mathrm{d}} \pm 0,9$ \\
$45-50$ & $48,72 \pm 1,50$ & $51,0^{\mathrm{e}} \pm 1,8$ & $39,91 \pm 1,20$ & $40,2^{\mathrm{e}} \pm 0,8$ \\
Moyenne & $47,4 \pm 1,42$ & $47,6 \pm 41,86$ & $38,65 \pm 1,08$ & $37,5 \pm 0,98$ \\
\hline
\end{tabular}




\section{DISCUSSION}

Les œufs de pintade originaires du Centre pèsent à $49 \%$ de cas entre 35 et $40 \mathrm{~g}$ alors que ceux du Sahel à $49 \%$ pèsent entre 41 et $45 \mathrm{~g}$. Ce résultat corrobore celui de Sanfo (2006a) qui a rapporté que le poids des œufs de pintade varie entre 20 et $50 \mathrm{~g}$ avec la grande proportion qui pèse entre 35 et $45 \mathrm{~g}$. $\mathrm{Au}$ vu de ces chiffres, nous sommes amenés à dire que les œufs de Sahel sont plus lourds que ceux du Centre, et que la pintade du Sahel justifie bien son qualificatif de "grosse". La grande variabilité du poids des oufs de la pintade de la région Sahélienne du Burkina Faso offre une opportunité pour le tri des oufs destinés à la couvaison.

Les œufs du Sahel ont respectivement une longueur moyenne 47,4 $\pm 1,42 \mathrm{~mm}$ et un diamètre moyen de $38,65 \pm 1,08 \mathrm{~mm}$. Ceci est semblable aux mensurations des œufs de pintade du Centre qui présentent les longueurs moyens de 47,00 $\pm 1,9 \mathrm{~mm}$ et 36,9 $\pm 0,9 \mathrm{~mm}$ de diamètre (Sanfo, 2006a). Le taux de mortalité des pintadeaux est très élevé au Burkina Faso. Le taux de mortalité enregistré dans la présente étude est très inférieur à ceux de Bako (2004), qui a rapporté des taux allant de $73 \%$ à $100 \%$, de Hien et al. (2000) qui ont révélé un taux de mortalité de $90 \%$ en milieu villageois. D'après ces mêmes auteurs, les mortalités sont plus élevées aux mois de juillet (22\%), d'août (54\%) et de septembre (21\%). Les plus faibles taux de mortalité étant enregistrés aux mois d'octobre (1\%), de mai (2\%) et de juin (2\%) qui marquent le début de la saison pluvieuse (Savadogo, 1995). L'importance de cette mortalité est également soulignée par (Sanfo, 2007) qui rapportent les taux de $73 \%$ et $80 \%$ enregistrés dans des élevages améliorés et traditionnels. Selon ces auteurs, les causes de cette forte mortalité sont multifactorielles, incluant l'hygiène, les infections microbiennes, l'âge et la saison d'éclosion du pintadeau. Au titre de la reproduction, la ponte saisonnière et les faibles capacités des producteurs à incuber les œufs, doublées des fortes mortalités des pintadeaux affectent profondément la productivité numérique et réduisent de ce fait les pouvoirs financiers des producteurs.

Le poids des pintadeaux à l'éclosion $(25,7 \pm 2,8 \mathrm{~g})$ est similaire à celui trouvé par Sanfo (2006b). La courbe de croissance est également similaire à celle de (Sanfo, 2008) où les courbes des mâles et des femelles sont restées similaires jusqu'à la deuxième semaine. Par la suite, les femelles ont pris de l'ascendance sur les males jusqu'à la fin de l'étude (Sanfo, 2008).

La pintade locale est connue pour sa faible vitesse de croissance pondérale (Hien et al., 2000). Le GMQ de 2,5g est similaire à celui de 2,6 g/jour en système amélioré avec des aliments industriels, pendant la phase démarrage en milieux villageois (Bonkoungou, 2005). Le même auteur a noté un GMQ de 5,5 g/jour avec des pintadeaux nourris à l'aide d'aliments industriels pendant la phase croissance (au-delà de deux semaines). Ce qui est identique à celui trouvé dans cette étude qui est de 5,00 g.

\section{Conclusion}

Les œufs du Sahel semblent plus lourds que leurs homologues du Centre au vu de la ccomparaison des pourcentages du poids des œufs par catégorie. L'habitat, l'éleveuse la lampe tempête et le saccharose ont réduit considérablement les taux de mortalité des pintadeaux chez les trois producteurs, entrainant une moyenne de $8,63 \%$. Les poids à la naissance des pintadeaux mâles et femelles ont été les mêmes au moment de leur éclosion. Le poids des femelles a marqué une supériorité sur celui des mâles au cours de leur évolution. La vitesse de croissance des pintadeaux est restée faible avec le temps.

\section{REMERCIEMENTS}

Nous remercions l'Unité Economique des Etats de l'Afrique de l'Ouest (UEMOA) à travers le Projet d'Appui à l'Enseignement Supérieur (PAES) pour sa contribution financière. Sans elle, ce projet d'article n'aurait certainement pas vu le jour. 


\section{REFERENCES}

Bako A. 2004. Stratégies d'élevage de pintadeaux dans la zone de BoboDioulasso: pertes économiques et moyens paysans de réduction de la mortalité. Rapport ATE. Ouagadougou. Burkina Faso, p. 42.

Bonkoungou GFX. 2005. Characteristics and performance of guinea fowl production under improved and scavenging condition in the Sahelian Region of Burkina Faso. MSc, Thesis. The Royal Veterinary and Agricultural University. Copenhagen. Denmark, p. 59.

Gendron M, Blentz G. 1970. La qualité de l'œuf de consommation. Supplément aux Nouvelles de l'Aviculture. $\mathrm{N}^{\circ} 125$. Paris8è. France, p. 28.

Hien OC, Boly H, Diarra B, Sawadogo L. 2000. Influence du mode d'élevage sur la mortalité et la croissance des pintades en saison hivernale dans la zone SubHumide du Burkina Faso. Bull. Anim. Hlth. Prod. Afr, 48(3): 236-245.

Kaboret Y, Bessin R, Boussini H, Nagalo B. 2002. Mortalité de pintadeaux en élevage rural au Burkina Faso; approche étiologique. Rév. Etudes et Recherches Sahéliennes, 7(5): 16 22.
MRA. 2003. Ministère des Ressources Animales. Méléagriculture au Burkina Faso: Diagnostic et axes d'amélioration. Secrétariat Général. Burkina Faso, p. 46.

Sanfo R, Boly H, Sawadogo L, Brian O. 2006a. Caractéristiques physiques de l'œuf de la pintade locale (Numida meleagris) dans la région centre du Burkina Faso. Revue Sciences et Techniques. Burkina Faso, 48(4): 236-245.

Sanfo R, Boly H, Sawadogo L, Brian O. 2008. 2006b. Performances de ponte et caractéristiques des œufs de la pintade locale (Numida meleagris) en système de conduite améliorée dans la région Centre du Burkina Faso. Revue Élev. Méd. Vét. Pays Trop., 61(2) : 135-140.

Sanfo R. 2007. Paramètres de Reproduction de la Pintade Locale au Burkina Faso. Université de Ouagadougou : Ouagadougou, Burkina Faso ; 129.

Savadogo A. 1995. Contribution à l'amélioration de l'élevage de la pintade (Numida meleagris) au Burkina Faso. Mémoire de fin d'études d'IDR. Option: Elevage. Université de Ouagadougou. Burkina Faso, p.102. 